\title{
Iridocorneal endothelial syndrome
}

INSERM

\section{Source}

INSERM. (1999). Orphanet: an online rare disease and orphan drug data base.

Iridocorneal endothelial syndrome. ORPHA:64734

Iridocorneal endothelial (ICE) syndrome describes a group of progressive corneal proliferative endotheliopathies comprised of Chandlers syndrome, Cogan-Reese syndrome and essential iris atrophy (see these terms), affecting mainly young adult females and characterized by iris holes and atrophy, papillary distortion, anterior synechiae, corneal edema and often with secondary glaucoma and corneal decompensation as complications 